\title{
Protein-losing enteropathy after infectious enterocolitis in child - a case report and a review of the literature
}

\author{
Lorena Elena Melit ${ }^{1,2}$, Cristina Oana Marginean ${ }^{1,2}$, Andreea Dinca1, \\ Raluca Damian ${ }^{3}$ Cristian Dan Marginean² \\ ${ }^{1}$ Pediatrics Clinic 1, Targu Mures, Romania \\ ${ }^{2}$ University of Medicine and Pharmacy, Targu Mures, Romania \\ ${ }^{3}$ Neonatology Clinic 1, Targu Mures, Romania
}

\begin{abstract}
Protein-losing enteropathy (PLE) represents an abnormal protein loss at the level of the digestive tract mucosa, being in most of the cases secondary to other gastrointestinal or extraintestinal pathologies. Nevertheless, there also are primary causes, genetic ones associated with PLE. We present the case of a small female child, 1 year and 1 month-old, with a history of infectious enterocolitis determined by Escherichia coli enteropathogenic, who presented palpebral edemas, especially in the morning for approximately 1 week. The laboratory tests revealed hypoalbuminemia, type A and G hypogammaglobulinemia, leukocytosis with eosinophilia, and anemia. The test for the detection of occult hemorrhages from feces was positive, likewise the fecal calprotectin and level of alpha- 1 antitrypsin. We identified also allergy to cow's milk protein, which we interpreted as being secondary to the intestinal pathology. Thus, we established the diagnosis of proteinlosing enteropathy after an episode of acute enterocolitis caused by E. coli enteropathogenic, and we administered substitutive treatment with human albumin and steroids, with favorable evolution. The particularity of the case consists in diagnosing a severe case of protein-losing enteropathy in a small child, with palpebral edema, predominantly during the morning, and decreased levels of serum albumin, after an episode of acute enterocolitis caused by E. coli enteropathogenic, which remitted without etiologic treatment.
\end{abstract}

Keywords: protein-losing enteropathy, enterocolitis, Enteropathogenic Escherichia coli, child
Abbreviations
Alb - albumin
CBC - complete blood
IgA - immunoglobulin A
CT - computer tomography
IgG - immunoglobulin G
PLE - protein-losing enteropathy
Hb - hemoglobin
Eo - eosinophils
Htc - hematocrit
Leu - leucocytes
E. coli - Escherichia coli
W - weight

\section{INTRODUCTION}

Protein-losing enteropathy (PLE) is represented by an abnormal protein loss at the level of the digestive tract that leads to a decreased of serum level proteins. In most of the cases, it appears as a secondary manifestation associated to other gastrointestinal or extraintestinal pathologies (1). The pathological mechanism of
PLE consists either in protein loss by the existence of some erosions or ulcerations of the mucosa, either by impaired protein absorption due to the inflammation of the intestinal mucosa. The causes that can produce erosions or ulcerations of the mucosa can be infectious, such as: acute diarrhea, infection with Clostridium difficile, Clostridium perfringens, Cytomegalovirus, 
Helicobacter pylori, infestation with Giardia lamblia or Strongyloides stercoralis; or non-infectious: allergic gastroenteritis, ulceration or ischemia after enteric anastomosis, celiac disease, gastrointestinal tumors, host versus graft disease, Henoch-Schonlein purpura, intestinal inflammatory diseases, multiple polyposis, portal hypertension, newborn's necrotizing enterocolitis, systemic lupus erythematosus (1). On the other hand, another entity that can cause PLE is intestinal lymphangiectasis: primary lymphatic obstruction (familial, Noonan syndrome) or secondary (arsenic poisoning, cardiac failure, constrictive pericarditis, injuries of the thoracic duct, inflammations, retroperitoneal tumors, vascular thromboses after liver transplantation) (1). There have also been described certain innate metabolism disorders that are associated with PLE: enterocyte deficiency in heparan sulfate by the lack of synthesis and glycosylation congenital disorders (1). The clinical manifestations are variate and depend on both, the degree of protein loss and the underlying pathology. The most frequent manifestation is diarrhea, but in cases where the protein loss is increased or prolonged, in severe cases, peripheral edemas or ascites can also appear secondary to oncotic pressure decrease. The laboratory tests will point out decreased levels of serum proteins, such as albumin, alfa- 1 antitrypsin, ceruloplasmin, fibrinogen, transferrin, but also hypogammaglobulinemia or lymphopenia. Other investigations useful in order to establish the diagnosis are the imagistic ones, such as enema with barium, video-capsule exam or even abdominal CT. The esophagogastroduodenoscopy with biopsies from the digestive mucosa is the one that established in most of the cases the etiologic diagnosis of PLE. The diagnosis of PLE can be established also by non-invasive methods, such as the determination of alfa- 1 antitrypsin and calprotectin from feces $(2,3)$. The management of PLE must be always focused on the treatment of the underlying disease. Never- theless, in severe cases, the administration of a supportive therapy is also needed, such as human albumin, antibiotics, or even probiotics. The evolution and prognosis of patients diagnosed with protein-losing enteropathy depends mostly on the adequate treatment of the underlying disease and its evolution.

\section{CASE PRESENTATION}

We present the case of a 1 year and 1 monthold female child, admitted in our clinic with palpebral edemas, especially during the morning, from approximately 1 week. Her personal history revealed an episode of acute diarrhea approximately 6 weeks before the present admission, and the stool culture was positive for E. coli enteropathogenic, but the symptoms disappeared without treatment. The actual disease had the onset approximately 1 week ago with palpebral edemas especially in the morning, interpreted by the general practitioner in context of an ocular pathology, reasons for which he recommended local treatment, but without any sign of improvement.

The clinical exam in the moment of admission revealed the following pathological elements: pale skin, mild palpebral edemas and also, edemas of the inferior limbs bilaterally, W: $12 \mathrm{~kg}$.

The laboratory tests showed hypoalbuminemia (Alb: $2.31 \mathrm{~g} / \mathrm{dL}$ ), hypocalcemia (2.13 mmol/L), hypogammaglobulinemia type $\mathrm{A}$ and G (IgA: $19 \mathrm{mg} / \mathrm{dL}$, IgG: $146 \mathrm{mg} / \mathrm{dL}$ ), but also a decreased level of total proteins $(3.53 \mathrm{~g} / \mathrm{dL})$. The CBC count pointed out leukocytosis (Leu 14 $740 / \mu \mathrm{L}$ ), anemia (Hb $10.3 \mathrm{~g} / \mathrm{dL}, \mathrm{Htc} 30.8 \%$ ), and eosinophilia (Eo $1630 / \mu \mathrm{L}$ ). The inflammatory biomarkers, namely the erythrocyte sedimentation rate and $\mathrm{C}$ reactive protein were in normal limits. The peripheral smear proved the presence of an increased percentage of eosinophils, $9 \%$, but also of lymphocytes, of $64 \%$. We raised the suspicion of a celiac disease, which was infirmed based on the negative serology. We identified an increased level of alfa- 1 antitrypsin in 
the feces (1 $846 \mu \mathrm{g} / \mathrm{g})$, and also of fecal calprotectin (1 $183 \mu \mathrm{g} / \mathrm{g})$. Due to the presence of eosinophilia, we performed also a panel of pediatric specific allergens, pointing out allergy to cow's milk proteins, but we interpreted it as being secondary to the intestinal inflammation. The test for occult hemorrhages from stools was positive, and the digestion tests pointed out multiple fat drops, but also rare crystals of fatty acids. The urinary exam, the stools culture and the exam for parasites were negative. We also performed an abdominal ultrasound, but without pathological elements.

Thus, we established the diagnoses of protein-losing enteropathy after an episode of acute enterocolitis caused by E. coli enteropathogenic and allergy to cow's milk proteins. The administered treatment consisted in human albumin associated with intravenous steroids for 5 days, with the normalization of albumin and total serum proteins. Due to the detection of occult hemorrhages in stools, we decided also to administer a drug with bacteriostatic role (biseptol) for 5 day, and also probiotics for 1 month. The diet management involved a restrictive diet with the elimination of all dairy products and the initiation of a dietetic milk formula. The recommendations at the moment of discharge consisted in the continuation or oral steroids (prednisone) for one month associated with proton pump inhibitors, aspacardin and calcium supplements, but also alimentation with dietetic milk formula and sparing diet without dairy products. After approximately one month, the patient's evolution was favorable, with laboratory parameters in normal ranges, and without occult hemorrhages in stools.

The particularity of the case consists in diagnosing a severe case of protein-losing enteropathy in a small child, with palpebral edema, predominantly during the morning, and decreased levels of serum albumin, after an episode of acute enterocolitis caused by E. coli enteropathogenic, which remitted without etiologic treatment.

\section{DISCUSSIONS}

Protein-losing enteropathy represents in most of the cases a syndrome of a gastrointestinal disorders, while primary intestinal lymphangiectasis or Waldmann disease as a cause of this entity, is much rarer encountered (4). Multiple infectious or non-infectious gastrointestinal or extraintestinal pathologies can cause PLE. One of the severe pathologies, with reserved prognosis, that can cause PLE, is colon cancer. Thus, Hayashi described the case of a 29 year-old young female that presented with severe edemas of the inferior limbs, having multiple polyps of the stomach and colon, but also multiple tumors in the liver, being diagnosed with familial adenomatous polyposis and colon cancer with hepatic metastases (5). The albumin serum level in this case was $1.1 \mathrm{~g} / \mathrm{dl}$, while in our case it was $2.31 \mathrm{~g} / \mathrm{dl}$. Also, our patient presented with palpebral edema in the morning and not of the inferior limbs, probably also due to the small age. Nevertheless, the clinical exam showed also in our case mild edemas of the inferior limbs, but we mention that the patient was examined in the afternoon, fact that could explain the edemas in the lower part of the body. Paraneoplasic PLE can also appear in case of other types of tumors, such as lymphomas. Thus, Stanek et al described the case of a 47 year-old male patient, with hypoalbuminemia and ankle edemas in his history, who presented with chronic diarrhea and abdominal pain, and was diagnosed with marginal zone lymphoma and PLE, without presenting ulceration of the digestive mucosa. In this case, the prolonged treatment with steroids did not present any impact on the symptomatology, the disappearance of diarrhea and edemas occurring only after chemotherapy and tumor remission (5). Therefore, the treatment of the underlying disease in case of a patient diagnosed with PLE, presents a major role. In case of our patient, the treatment with steroids had an essential role due to the fact the PLE was the result of on inflammation of the intestinal mucosa. 
Another case diagnosed with PLE at approximately the same age as our patient, is the one of a 22 month-old child, known since birth with type I diabetes mellitus caused by a pancreatic hypoplasia, in whom the abdominal MRI exam pointed out a minimal quantity of ectopic pancreatic tissue, but also the lack of the gallbladder and hepatic steatosis, and the exam with videocapsule pointed out diffuse erythema and ulcerations of the gastric mucosa, and edema of the short bowel mucosa. In this case, a clear relationship between the chronic pathology and the development of PLE could not be established. The patient's clinical evolution was favorable under treatment with steroids and enoxaparin (6). Our patient also presented favorable evolution, with the remission of palpebral edemas and normalization of the serum albumin after substitutive treatment with albumin for 5 days and steroids treatment for 4 weeks. In certain cases, PLE, can be manifested also by pleural effusion and ascites, when hypoalbuminemia is severe. Thus, it was reported the case of a 30 year-old female, diagnosed with Sjögren syndrome and PLE, presenting also severe edema, with favorable evolution under oral steroids and hydroxychloroquine (7). Other infrequent causes of PLE are congenital malformations of the lymphatic vessels and retroperitoneal fibrosis, like in the case described by Mahjoub et al, of a 2 year and 5 month-old female child, with mesointestinal fibrosis (8). Collagenous colitis is another rare intestinal pathology that can lead to PLE in small ages, with favorable evolution under steroids treatment (9).

Bacteria, viruses and parasites are among the infectious causes of PLE. Thus, it was described in the literature, the case of a 52 year-old female with chronic diarrhea and anasarca, whose biopsies from the duodenal mucosa pointed out the presence of Giardia lamblia, with the remission of edemas and diarrhea after anti-helminthic treatment (10). Among the cases of PLE determined by viral infections, were reported in the literature 8 cases of infection with cytomegalovirus associated with PLE (11), but also a case of PLE after an episode of acute gastroenteritis determined by Rotavirus in a 6 month-old infant (12). Similarly, in the case presented by us, the patients presented PLE after an episode of acute infectious enterocolitis caused by E. coli enteropathogenic.

PLE can also appear as a result of a non-organic, external cause, like in the case described by Altaf of a 1 year and 8 month-old child, diagnosed with allergic enterocolitis and PLE after an accidental ingestions of an accumulator, as an effect of manganese on the intestinal mucosa (13).

The management of PLE involves mainly the treatment of the underlying disease, but includes also dietetic interventions, such as increased protein and low fat intake, but also supplementation with medium chain triglycerides (4).

\section{CONCLUSIONS}

Protein-losing enteropathy is in most of the cases secondary to other gastrointestinal or extraintestinal pathologies. Therapy with steroids is usually useful, but the treatment of the underlying disease remains the main goal in the management of PLE.

Conflict of interest: none declared Financial support: none declared

\section{REFERENCES}

1. Sylvester F.A. Protein-losing enteropathy. In Wyllie R, Hyams JS. Pediatric Gastrointestinal and Liver Disease. Elsevier, 2006:507-515.

2. Thomas D.W., Sinatra F.R., Merritt R.J. Random fecal alpha-1-antitrypsin concentration in children with gastrointestinal disease. Gastroenterology 1981; 80:776-782.

3. Bunn S.K., Bisset W.M., Main M.J., Gray E.S., Olson S., Golden B.E. Fecal calprotectin: validation as a noninvasive measure of bowel inflammation in childhood inflammatory bowel disease. J Pediatr Gastroenterol Nutr 2001; 33:14-22.

4. Amiot A. Protein-losing enteropahy. Rev Med Interne 2015; 36(7):467-473. doi: 10.1016/j.revmed.2014.12.001 
5. Nadine Stanek, Peter Bauerfeind, Guido Herzog et al. Marginal Zone Lymphoma Complicated by Protein Losing Enteropathy. Case Rep Hematol 2016; 2016: 9351408.

6. McMillan T., Girgis R., Sellers E.A.C. Neonatal diabetes and protein losing enteropathy: a case report. Abstr. In BMC Med Genet 2016; 17: 32 .

7. Liao C.Y., Chien S.T., Wang C.C. et al. Sjögren's syndrome associated with protein losing gastroenteropathy manifested by intestinal lymphangiectasia successfully treated with prednisolone and hydroxychloroquine. Lupus 2015; 24(14):1552-1556. doi: 10.1177/0961203315596078.

8. Mahjoub F., Sani M.N., Tabari A.K., Monajemzadeh M., Zandieh S. An Unusual Cause of Protein Losing Enteropathy in a 2.5-Year-Old Girl: Meso-Intestinal Fibrosis. European J Pediatr Surg Rep 2014; 2(1):29-31.

9. Almadhoun O.F., Katzman P.J., Rossi T. Collagenous Colitis Associated with Protein Losing Enteropathy in a Toddler. Case Rep Gastrointest Med 2014; 2014: 209624.
10. Furtado A.K., Cabral V.L.R., Santos T.N. Giardia infection: Protein-losing enteropathy in an adult with immunodeficiency. World J Gastroenterol 2012; 18(19):2430-2433.

11. Megged O., Schlesinger Y. Cytomegalovirus-associated protein-losing gastropathy in childhood. Eur J Pediatr 2008; 167(11):12171220. doi: 10.1007/s00431-008-0791-1.

12. Iwasa T., Matsubayashi N. Protein-losing enteropathy associated with rotavirus infection in an infant. World J Gastroenterol 2008; 14(10):1630-1632.

13. Altaf M.A,. Goday P.S., Telega G. Allergic enterocolitis and protein-losing enteropathy as the presentations of manganese leak from an ingested disk battery: A case report. J Med Case Reports 2008; 2:286. 